Supporting Information for the Communication entitled:

\title{
Patterned Growth of Large Oriented Organic Semiconductor Single Crystals on Self-Assembled Monolayer Templates
}

Alejandro L. Briseno ${ }^{1}$, Joanna Aizenberg ${ }^{2}$, Yong- Jin. Han ${ }^{2}$, Rebecca A. Penkala ${ }^{2}$, Hyunsik Moon ${ }^{2}$, Andrew J. Lovinger ${ }^{2}$, Christian Kloc ${ }^{2}$, and Zhenan Bao ${ }^{3} *$

${ }^{l}$ Department of Chemistry and Biochemistry, University of California- Los Angeles, ${ }^{2}$ Bell

Laboratories - Lucent Technologies, and the ${ }^{3}$ Department of Chemical Engineering, Stanford University

zbao@chemeng.stanford.edu; jaizenberg@lucent.com

\section{Self-Assembled Monolayers Used}

$\mathrm{HS}\left(\mathrm{CH}_{2}\right)_{10} \mathrm{CO}_{2} \mathrm{H}$

Mercaptoundecanoic Acid

$-\mathrm{CO}_{2} \mathrm{H}$
$\mathrm{HS}\left(\mathrm{CH}_{2}\right)_{2} \mathrm{NH}_{2}$

Aminoethanethiol

$-\mathrm{NH}_{2}$
$\mathrm{HS}\left(\mathrm{CH}_{2}\right)_{11} \mathrm{CH}_{3}$

Dodecylthiol

$-\mathrm{CH}_{3}$
$\longrightarrow-\mathrm{SH}$

Thiophenol

$-P$

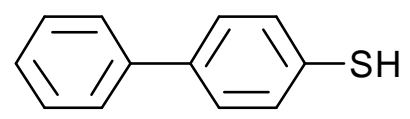

Biphenylthiol

$-2 P$

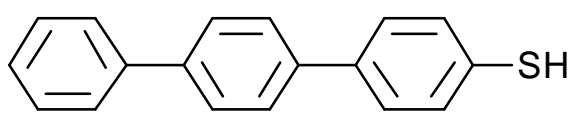

Terphenylthiol

$-3 P$

Organic Semiconductors Used<smiles>c1ccc2cc3ccccc3cc2c1</smiles>

Anthracene<smiles>Clc1c2ccccc2cc2cc3ccccc3cc12</smiles>

5-Chlorotetracene 
Materials. Tetrahydrofuran (THF, HPLC grade 99.9\%), methylene chloride (HPLC grade 99.9\%), ethanol (absolute 99.9\%), isopropanol, sulfuric acid, hydrogen peroxide (30\%), toluene, anthracene (99.9\%), and acetone were all purchased from Aldrich Chemicals and used as received except anthracene (recrystallized 4 times in toluene and washed with cold ethanol). Terphenylthiol (denoted as -3P), biphenylthiol (denoted as 2P) were synthesized as previously reported. ${ }^{1}$ Thiophenol (denoted as -P) was purchased from Aldrich chemicals and used as received. Sylgard 184 ${ }^{\mathrm{TM}}$ Polydimethylsiloxane (PDMS) was purchased from Dow Corning Chemicals $(1: 10 \mathrm{w} / \mathrm{w}$, against a cleaned lithographically patterned $\mathrm{Si}$ wafer and treated with Tridecafluoro-1,1,2,2tetrahydrooctyl)trichlorosilane $\mathrm{CF}_{3}\left(\mathrm{CF}_{2}\right)_{6}\left(\mathrm{CH}_{2}\right)_{2} \mathrm{SiCl}$ (Gelest, Inc.) to produce a non-stick interface. 11-mercaptoundecanoic acid (MUA), 2-aminoethanethiol hydrochloride, and dodecylthiol were purchased from Aldrich. Computer simulations of anthracene crystals were modeled using the software Shape 6.0 while molecules were drawn with ChemDraw Pro 8.0. Crystal structures of anthracene were viewed with the software Mercury 1.4

Self-Assembled Monolayers and Crystal Growth Conditions. (Note: this procedure does not include thiol-patterning of substrates and simply describes the method for templating the formation of organic single-crystal growth onto the different SAM-covered substrates). Substrates were prepared by evaporating $\mathrm{Au}(100 \mathrm{~nm})$ on a $\mathrm{Si}$ (100) wafer primed with $15 \mathrm{~nm}$ of $\mathrm{Ti}$ as an adhesion promoter. Gold substrates were cut into $2 \times 2 \mathrm{~cm}$ pieces and cleaned in a piranha (3:1 ratio of $\mathrm{H}_{2} \mathrm{SO}_{4}$ and $30 \% \mathrm{H}_{2} \mathrm{O}_{2}$ ) solution for 5 minutes, washed with deionized water, and dried with nitrogen. $-3 \mathrm{P}$ was prepared in a $2 \mathrm{mM}$ methylene chloride solution and refrigerated until further use. $-2 \mathrm{P},-\mathrm{P}$, and the remaining alkylthiol SAMs were prepared in $2 \mathrm{mM}$ ethanol solutions and refrigerated as well. Gold substrates were soaked faced-down in corresponding SAM solutions for at least 10 hours and no more than 24 hours. Substrates were washed in the respective SAM-prepared solvent, rinsed with isopropanol, and dried with nitrogen. The surface coverage of the SAMs were determined by reductive desorption using electrochemistry as described from literature (approximately 10-15 experiments were conducted for each thiol used in this study). ${ }^{2}$ The substrates were immersed in a saturated THF-anthracene 
solution in a test tube (parafilm-covered with punched holes), with the functionalized surface faced downward to avoid collecting any solution-borne crystals. The solvent was allowed to evaporate over a period of several days at a rate of $2 \mathrm{ml} / \mathrm{day}$ at room temperature. The solvent was not allowed to evaporate below the substrate level since a thin film crystalline film would form. Anthracene crystals typically form within 1-2 days of evaporation. The substrates are immediately transferred to an isopropanol solution and gently "dip-rinsed" several times (in separate alcoholic vials) to wash off any residual anthracene crystals. The samples were dried with nitrogen and placed in Fluoroware containers until further use. DCM was used as the solvent for growing 5-chlorotetracene (5-CT) crystals by the dip-coating method. Chloroform was also used to grow 5-CT crystals. No other solvents were used to grow crystals, but it should be possible to grow crystals in solvents that can dissolve the materials and as long as the vapor pressure of the solvent is relatively high. If the vapor pressure of the solvent is too low it will not be possible to dip-coat crystalline films and it will not be possible to grown crystals by slow evaporation.

\section{References.}

1. de Boer, B.; Martin M. Frank, M. M.; Chabal, Y. J.; Jiang, W.; Garfunkel, E.; Bao, Z. Langmuir 2004, 20, 1539-1542.

2. Satjapipat, M.; Raymond Sanedrin, M.; Zhou, F. Langmuir 2001, 17, 7637-7644. 


\section{Patterned Growth of Anthracene Crystals}

Solvent Evaporation Method. The crystals were grown under exact conditions as described from above, except the substrates are now thiol-patterned with SAMs. SAMs on gold were formed using microcontact printing. PDMS stamps with various relief structures were "inked" with a $2 \mathrm{mM}$ solution of $\mathrm{HS}\left(\mathrm{CH}_{2}\right)_{11} \mathrm{CH}_{3}$ in ethanol and brought into contact with the gold for no more than 10 seconds. The samples were rinsed with isopropanol, and subsequently immersed into -3P SAM for 30 minutes to derivatize the regions that were not stamped. The patterned substrates were carefully rinsed with isopropanol, dried with nitrogen and placed into a saturated solution of anthracene in the exact manner as described in the previous section.

Note: All Patterned domains below are 100x100 $\mu \mathrm{m}$
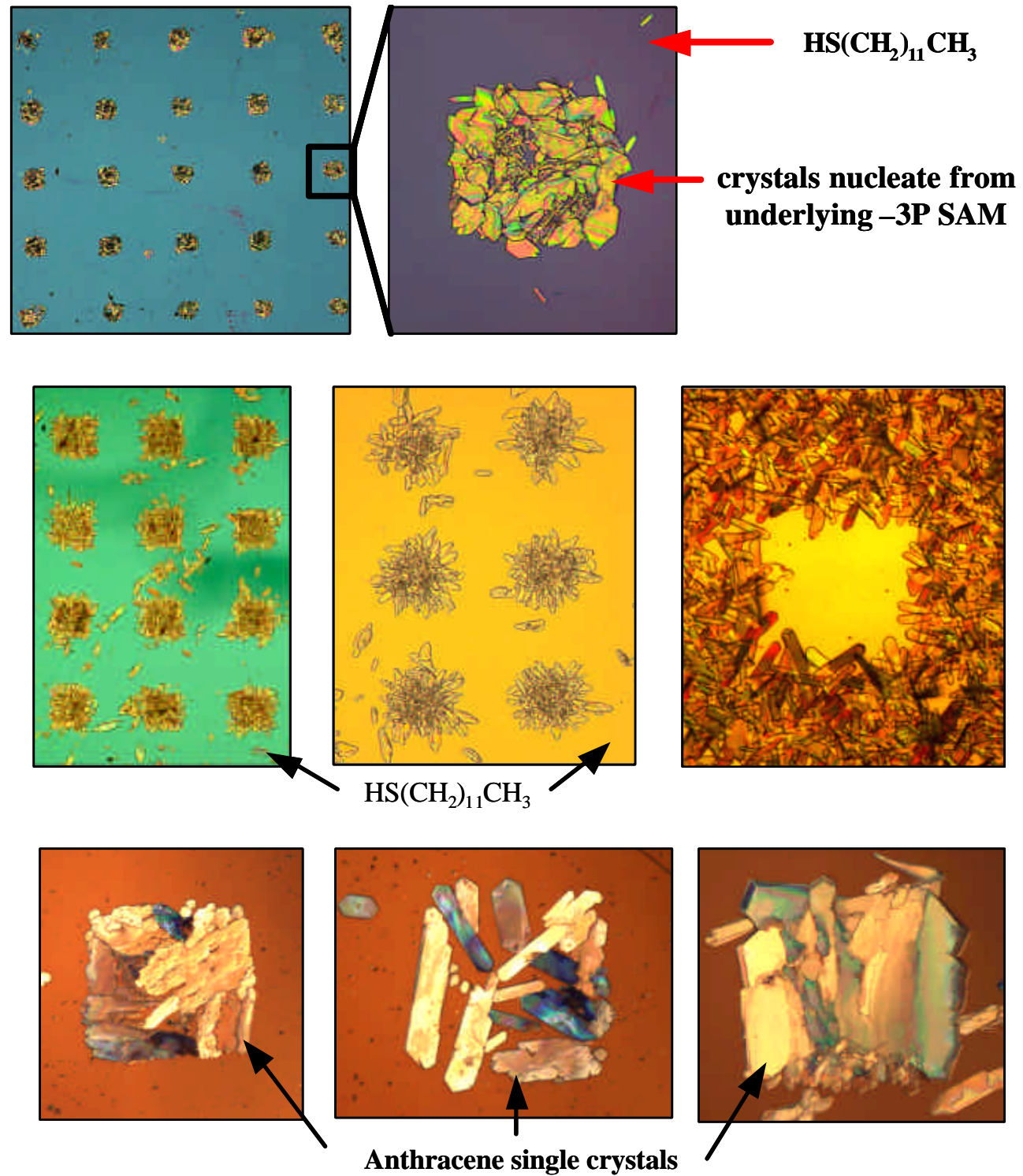
Dip-coating Method. Substrates were patterned as described above. The thiolpatterned substrates were placed on an alligator clip that was connected to a standard laboratory syringe pump that slowly pulled the substrate from a 10-fold diluted THF-anthracene solution (or $3 \mathrm{mg} / \mathrm{ml}$ in the case of 5-chlorotetracene in DCM). The anthracene solutions were prepared in quartz UV vials that were housed in an acrylic chamber with a sliding door for accessibility to samples. Humidity in the chamber was not monitored. If a saturated anthracene solution were instead used for pulling patterned films, a film would usually form instead of individualized domains containing crystalline films or singles crystals. Typical pulling rates were 11 to $110 \mu \mathrm{m} / \mathrm{sec}$.

\section{Anthracene Patterned Films:}

Crystalline films form on "patterned" underlying -3P SAM

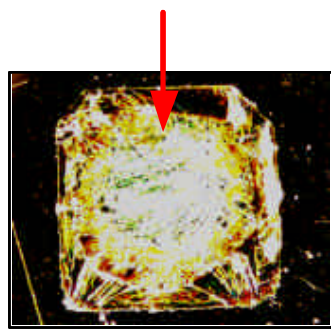

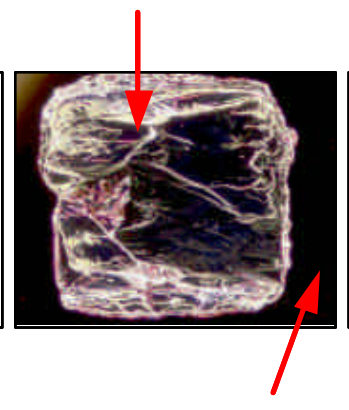

$\mathrm{HS}\left(\mathrm{CH}_{2}\right)_{11} \mathrm{CH}_{3}$
Crystalline films form on "patterned" underlying -3P SAM

\section{5-Chlorotetracene (5-CT) patterned Films:}

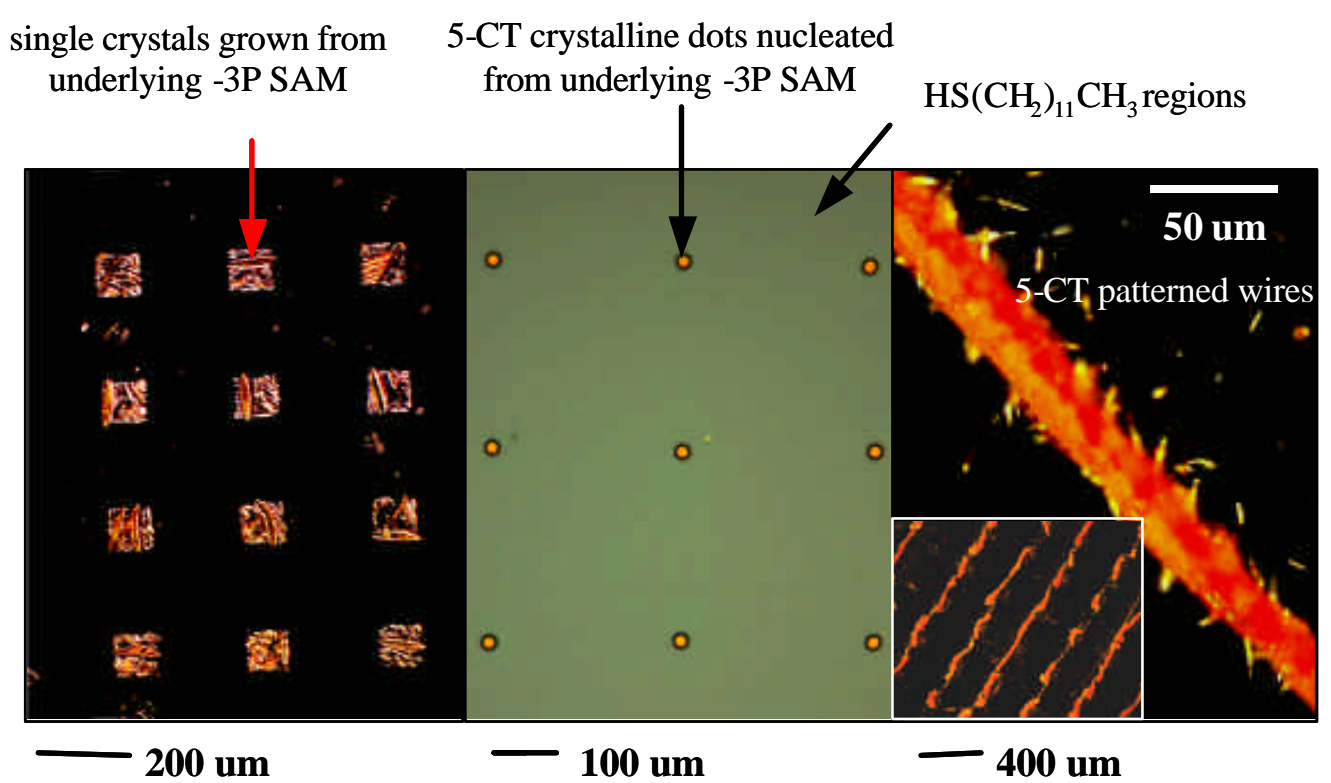




\section{Diagrammatic Illustration of Site-Specific Crystallization}

The cartoon below is not to scale and is only intended to illustrate a possible mechanism of crystal growth on SAM-patterned gold substrates. It can be seen that the flux of anthracene molecules to the growing crystals in the -3P SAM regions will induce nearsurface concentration gradients and deplete anthracene molecules from the less active alkylthiol areas. As a result, increased surface coverage and highly selective, patterned growth of oriented organic semiconductor single crystals are observed. A detailed mechanistic assessment has been reported for the high-resolution growth of inorganic crystals on patterned SAM templates. ${ }^{1}$

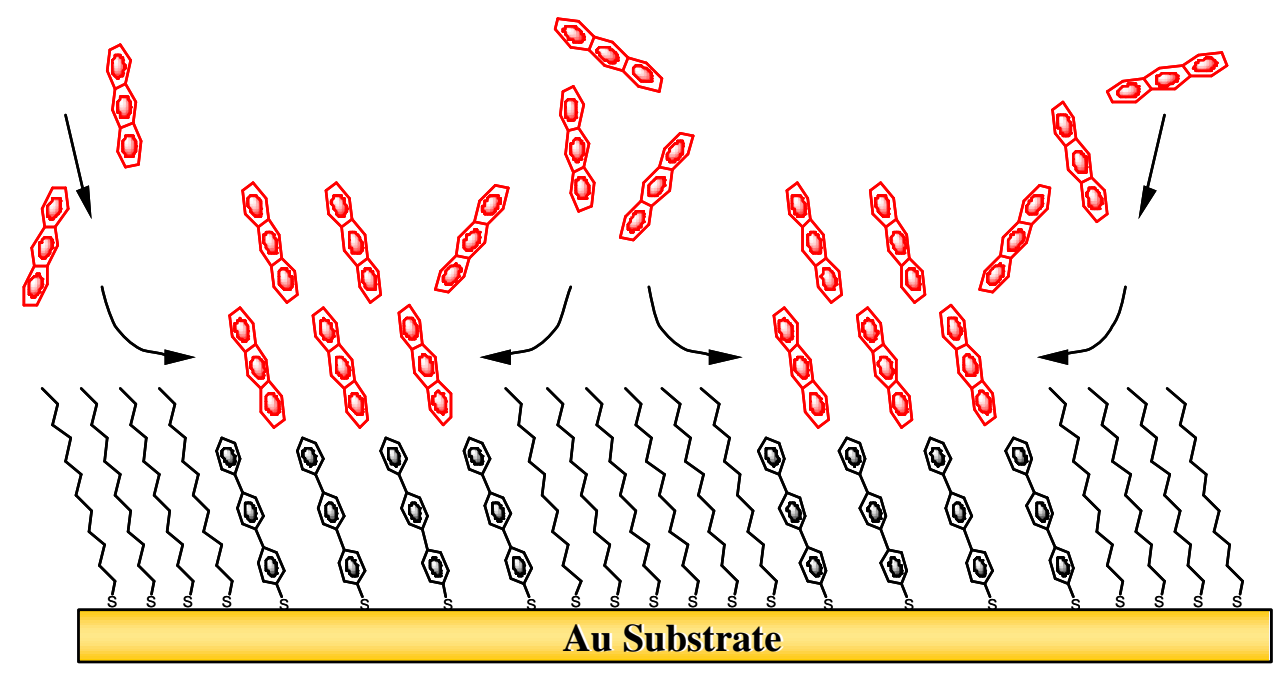

1. Aizenberg, J.; Black, A. J.; Whitesides, G. M. Nature 1999, 398, 495-498. 
Defining facet angles $\delta_{1}$ and $\delta_{2}$

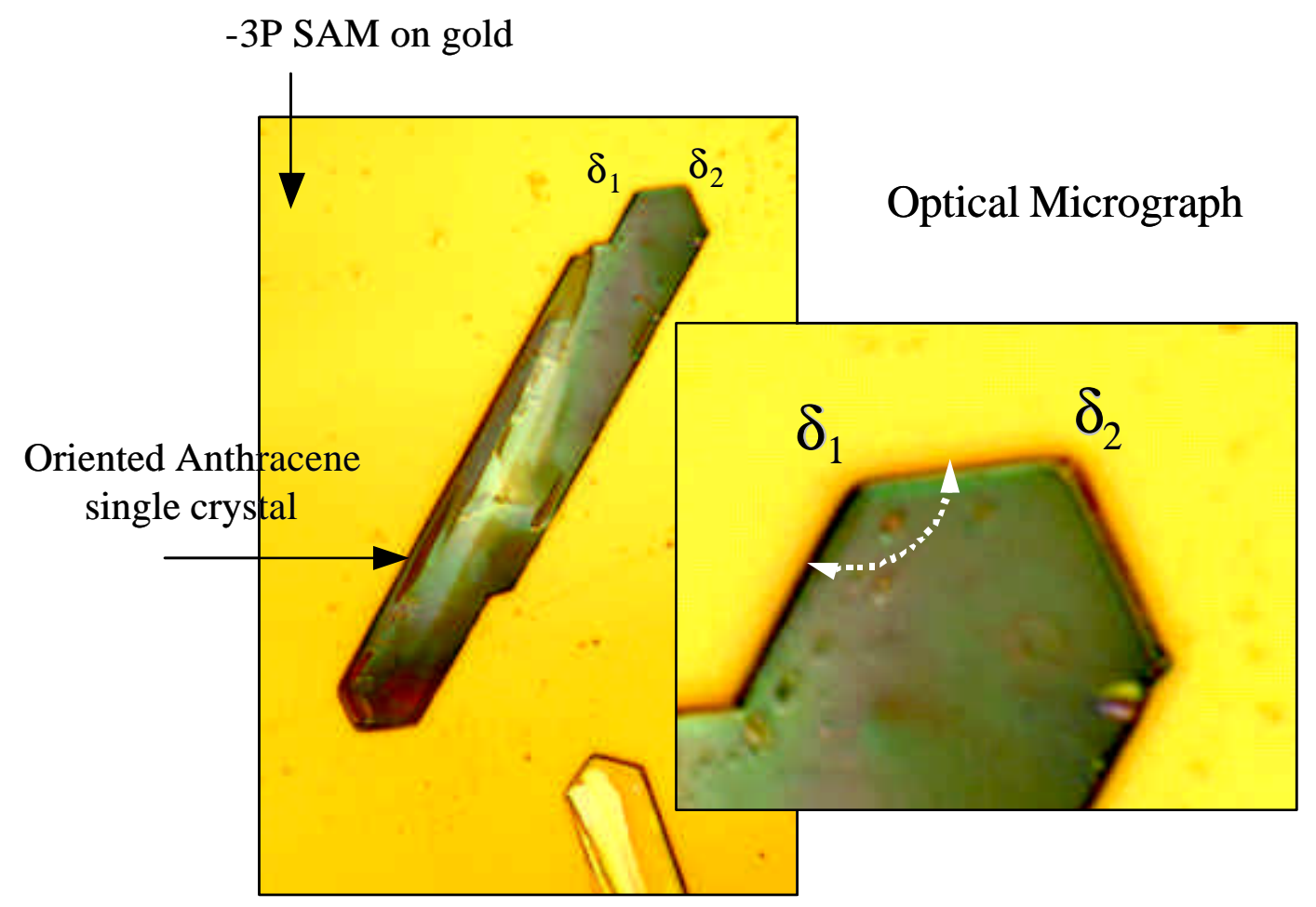

\begin{tabular}{|c|c|c|}
\hline Angle & $\begin{array}{c}\text { Experimentally } \\
\text { Measured }\end{array}$ & $\begin{array}{c}\text { Theoretically } \\
\text { Calculated }\end{array}$ \\
\hline$\delta_{1}$ & $125.8 \pm 2.6^{\circ}$ & $125^{\circ}$ \\
\hline$\delta_{2}$ & $109.7 \pm 4.0^{\circ}$ & $110^{\circ}$ \\
\hline
\end{tabular}




\section{XRD Pattern of Anthracene crystals grown on -3P passivated gold substrate}

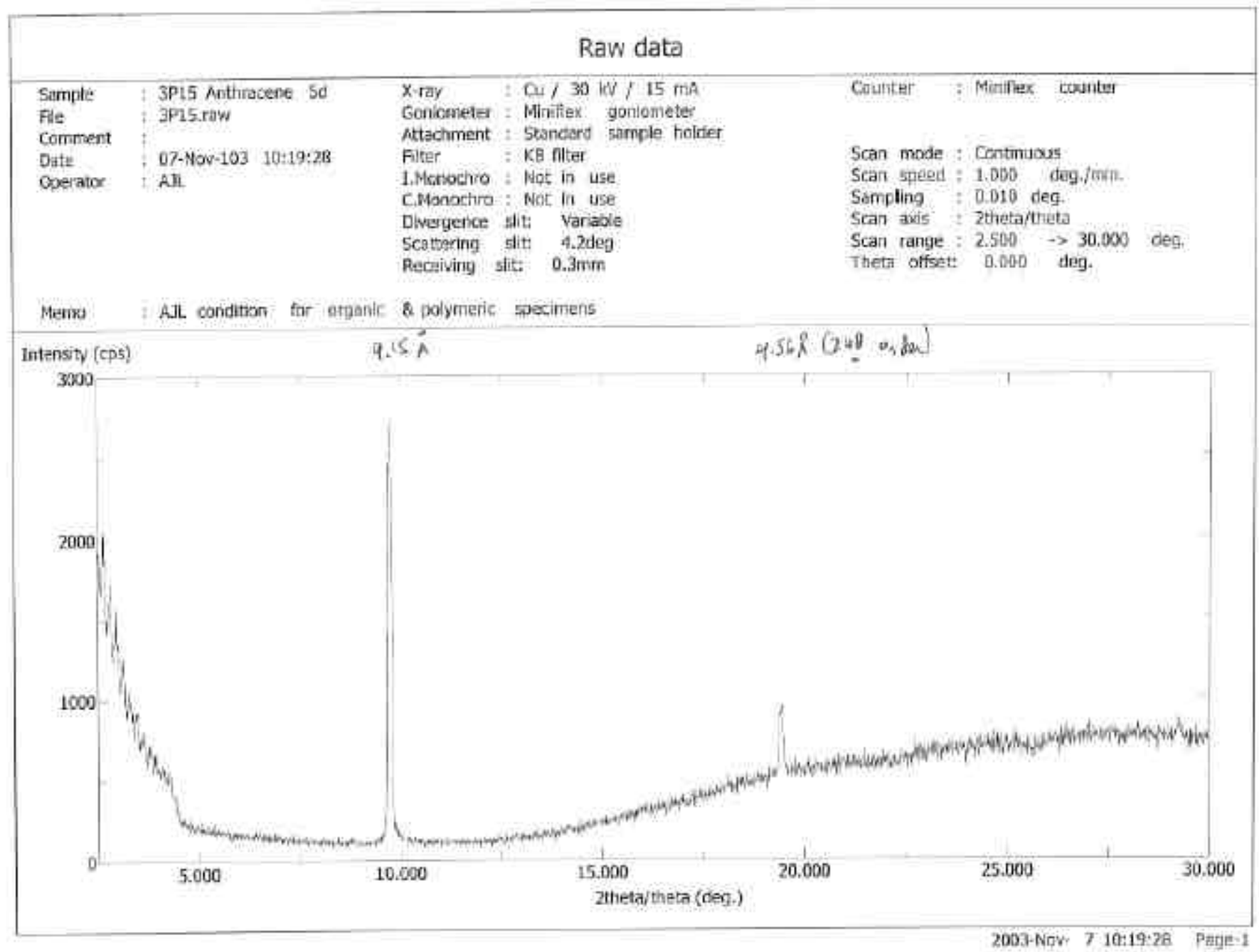




\section{Device Characteristics of Patterned Single Crystals}

Preliminary experiments show that functional transistors can be prepared with organic single crystals patterned in regions containing terphenylthiol $(-3 \mathrm{P})$. We extended our patterning method onto transistor devices by patterning dodecylthiol $\left(-\mathrm{CH}_{3}\right)$ and terphenylthiol (-3P) onto source-drain electrodes. 5 -chlorotetracene $(5-\mathrm{CT})^{1}$ crystals were selectively grown onto $-3 \mathrm{P}$ containing regions as shown below. Single crystal devices of the same material have been published with reported mobilities of $1.4 \times 10^{-4} \mathrm{~cm}^{2} / \mathrm{Vs}$. ${ }^{1}$ Our patterned transistors give average mobilities of $5.7 \times 10^{-4} \mathrm{~cm}^{2} / \mathrm{Vs}$ with a high of 0.003 $\mathrm{cm}^{2} /$ Vs. While the mobility is not as high as other single crystal results, it is, however, higher than the mobilities from single crystal devices of the same material (5-CT) from the previously reported work. Furthermore, our highest mobility attained is larger by more than one order of magnitude $\left(0.003 \mathrm{~cm}^{2} / \mathrm{Vs}\right.$ vs. $\left.1.4 \times 10^{-4} \mathrm{~cm}^{2} / \mathrm{Vs}\right)$. Therefore, this patterning method shows that patterned arrays of single crystals can be fabricated with mobilities consistent to those of known materials. Because further optimization of device structure and performance is still required, we are limited in providing additional device characteristics and more development is underway. Below we show a representative field-effect output characteristic corresponding to the highest mobility attained along with a representative micrograph of a single crystal of 5-CT grown on $-3 \mathrm{P}$ patterned sourcedrain electrodes (image does not correspond to output curve experiment). The image shows the nucleation of the crystal across the channel to yield a functional device.
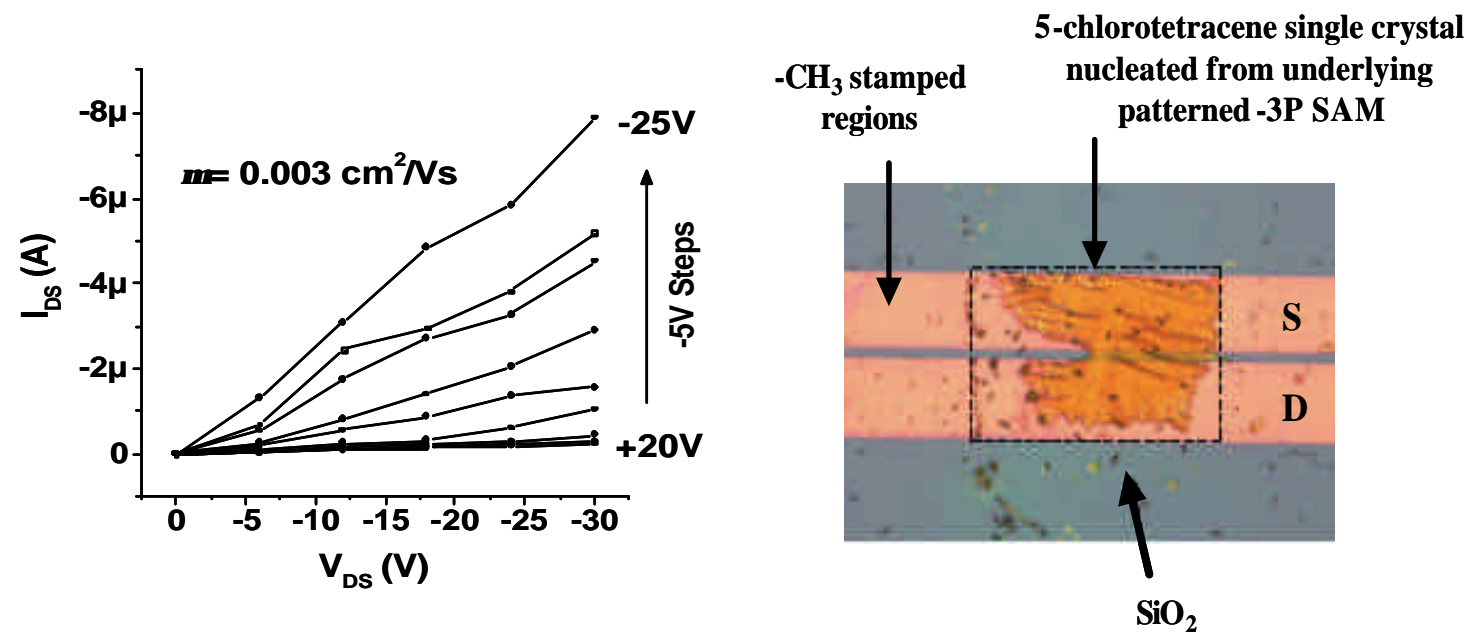

1. Moon, H; Zeis, R.; Borkent, E-J.; Besnard, C.; Lovinger, A.J.; Siegrist, T.; Kloc, Ch.; and Bao, Z. J. Am .Chem. Soc. 2004, 126, 15322-15323.

Acknowledgment. We gratefully acknowledge Dr. M. M. Ling and Mr. Ricky Tseng for their assistance with device measurements and helpful discussions 